\title{
Cyclic Spreading Codes for CDMA Systems
}

\author{
Jin Lu and Behnaam Aazhang \\ Laboratory for Communications Research \\ Dept. of Electrical and Computer Engineering \\ Rice University \\ Houston, TX 77251-1892, USA \\ Email jinlu@rice.edu, aaz@rice.edu
}

\section{Abstract -}

In this paper, we propose a CDMA system using cyclic convolutional matched filter. The multiple access interference in this system will be small under arbitrary relative delays.

\section{Cyclic Matched Filter and Cyclic Codes}

In deterministic CDMA systems, spreading codes of a user repeat in each bit interval, i.e., the code length is as long as the processing or spreading gain. Thus, observed in the span of one bit interval, say $[0, T]$, the codes with different delays correspond to certain cyclic shifts of the code itself, provided that the adjacent bits are identical (we will also consider the case in which the adjacent bits are different). Therefore, if we can design codes such that the cyclic convolutions of two different codes on $[0, T]$ are small, the cross correlation of different codes in the cyclic sense will also be small. That is, the multiple access interference will be small under arbitrary relative delays, when we use a cyclic matched filter detector.

The sufficient statistics of the cyclic MF is the same as a conventional MF. Therefore, in a multiuser scenario with an AWGN channel, it is suboptimal.

These codes for cyclic matched filter can be designed based on different performance criteria. One can minimize the average MAI over all users while keeping code bandwidth and bit energy fixed. Alternatively, one can maximize the average code bandwidth while keeping MAI and bit energy fixed. Since chip waveforms in this scheme are not necessarily rectangular, we define a spreading merit factor based on autocorrelation of codes to measure their bandwidths.

The time-consuming draw back of the above nonlinear constrained optimization problem is the main reason for searching suboptimal alternatives. To reduce the complexity in optimization computation, we solve the problem in two steps: 1 . Design meta-codes $c_{i}(l)$ 's with length $L$ for each user such that they are orthogonal to each other; 2 . With these meta-codes $c_{i}(l)$ 's, we then generate spreading codes $s_{i}(l)$ by extending $c_{i}(l)$ in different ways, i.e.

$$
s_{i}(l)=\sum_{n=0}^{N-1} a_{n}^{i} c_{i}(l-n L),
$$

where $\left\{a_{n}^{i}\right\}$ can be selected deterministically or randomly which may result in different code bandwidth.

\section{Numerical RESULT}

In this first design problem, we try to design unit energy codes with spreading merit factor $\mathcal{B}_{0}=3$ (corresponding to msequences). The autocorrelation and cross correlation of two such codes are in Figure-1. The average MAI of cyclic codes
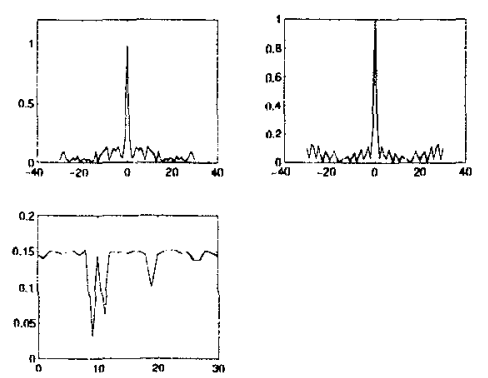

Figure 1: Autocorrelation and Cross Correlation (Optimal Design)

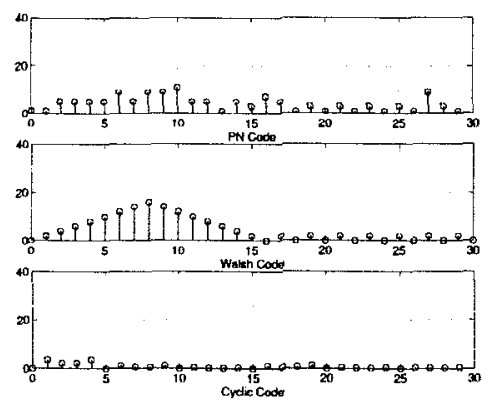

Figure 2: Cross Correlation (Suboptimal Design)

is 0.1529 , while for $\mathrm{m}$-sequences of the same length $(N=31)$ it is 0.3548 .

In the second design problem, we design the cyclic codes with the suboptimal scheme. The cross correlation of PN code, Walsh code and cyclic code with non-identical adjacent bits are shown in Figure-2. The cross correlation of cyclic codes is observed to be much better.

\section{REFERENCES}

[1] Naoki Suehiro, "A Signal Design without Co-channel Interference for Approximately Synchronized CDMA Systems", IEEE Journal on Selected Areas in Communications, Vol 12, No 5, June 1994.

[2] G.W.Wornell, "Spread-Response Precoding for Communication over Fading Channels", IEEE Trans. on Information Theory, Vol 42, No 2, pp.488-501, March 1996. 